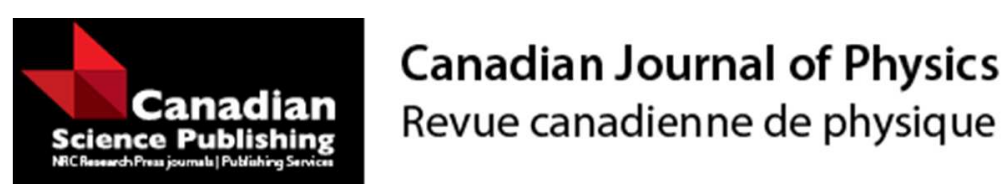

\title{
FLRW Cosmology of Induced Dark Energy Model and Open Universe
}

\begin{tabular}{|c|c|}
\hline Journal: & Canadian Journal of Physics \\
\hline Manuscript ID & cjp-2016-0827.R3 \\
\hline Manuscript Type: & Article \\
\hline Date Submitted by the Author: & $11-A p r-2017$ \\
\hline Complete List of Authors: & Bahrehbakhsh, Amirfarshad; Payame Noor University, Physics \\
\hline Keyword: & $\begin{array}{l}\text { Induced Matter Theory, FLRW Cosmology, Dark Energy, Geometry of the } \\
\text { Universe, Acceleration of Universe }\end{array}$ \\
\hline $\begin{array}{r}\text { Is the invited manuscript for } \\
\text { consideration in a Special } \\
\text { Issue? : }\end{array}$ & $\mathrm{N} / \mathrm{A}$ \\
\hline
\end{tabular}

\section{SCHOLARONE \\ Manuscripts}




\title{
FLRW Cosmology of Induced Dark Energy Model and Open Universe
}

\author{
Amir F. Bahrehbakhsh* \\ Department of Physics, Faculty of Science, Payam-e-Noor University, Iran
}

April 11, 2017

\begin{abstract}
We investigate the FLRW type cosmology of the Induced Dark Energy model and illustrate that the extra terms emerging from the fifth dimension can play the role of dark energy. The model predicts the expansion with deceleration at early time and acceleration in late time for an open universe.
\end{abstract}

Keywords: Induced-Matter Theory; FLRW Cosmology; Dark Energy; Geometry of the Universe.

\section{Introduction}

Observations of distant type Ia-supernovas indicate that universe is currently accelerating in its expansion [1]- [4]. Therefore, in a school of thought, the universe should mainly be filled with what usually is called dark energy. To explain this acceleration, a considerable amount of work has been performed in the literature [5]- [12].

The first and simplest explanation for dark energy is vacuum energy which, was introduced for the first time, by Einstein as cosmological constant to explain static universe. However, after Hubble demonstration of the universe expansion, Einstein called the cosmological constant his biggest blunder, but this term is preserved today to explain the current acceleration of the universe expansion [5]. The cosmological constant has perfect fit with observations and has equation of state parameter, $w_{\Lambda}=-1$. Though, it suffers two cosmological problems; The theoretical expectation of dark energy density value is 120 orders of magnitude bigger than the observations and more over, dark energy density and all matter (the baryonic and dark) energy density are of the same order today. Hence, many other models involving non-constant dark energy have been proposed, such as the quintessence [13]- [16], the k-essence [17, 18] and the chaplygin gas $[19,20]$ models. However, in most of them presuppose minimally coupled scalar fields with different priori added by hand potentials which, their origins are not clearly known.

Recently, some efforts based on the Brans-Dicke theory in which, the scalar field is non-minimally coupled to the curvature, has been performed to explain today's accelerating expansion of the universe $[21]-[26]$.

On the other hand, most of the attempts for geometrical unification of gravity with other interactions are based on using higher dimensions addition to our conventional four-dimensional $(4 D)$ space-time. After Nordstrøm [27], who was the first established a theory based on extra dimensions, Kaluza [28] and Klein [29] built a five-dimensional $(5 D)$ version of general relativity (GR) in which electrodynamics rises from an extra fifth dimension. After that, an intensive amount of works have been focused on this regard either via different mechanism for compactification of extra dimension

*email: amirfarshad@gmail.com 
or generalizing it to non-compact scenarios [30] such as the space-time-matter (STM) or inducedmatter (IM) theory [31, 32] and the Brane World scenarios [33]. The significant of the IM theory is that inducing $5 D$ field equations without matter sources leads to the $4 D$ field equations with matter sources.

In this work, following the Induced Dark Energy (IDE) model introduced in Ref. [34], for a $5 D$ version of GR, with generalized $5 D$ Friedmann-Lemaitre-Robertson-Walker (FLRW) metric, we investigate the cosmological implications of the model for an open universe. For this purpose, in the next section, with a brief review of the IDE model, we rewrite the cosmological equations and focus on the case of an open universe. We also provide a table and a figure for a better view of the deceleration and acceleration epoches of the universe expansion, which specify by the model. Finally, in the Sec. 3 conclusion is presented.

\section{FLRW cosmology of Induced Dark Energy model}

According to the Ref. [34] we consider $5 D$ version of the GR action as

$$
S=\int \sqrt{\left|{ }^{(5)} g\right|}\left(\frac{1}{16 \pi G}{ }^{(5)} R+L_{m}\right) d^{5} x,
$$

where, $c=1,{ }^{(5)} R$ is $5 D$ Ricci scalar, ${ }^{(5)} g$ is the determinant of $5 D$ metric $g_{A B}$ and $L_{m}$ represents the matter Lagrangian. Therefore, the Einstein field equations in five dimension will be

$$
{ }^{(5)} G_{A B}=8 \pi G{ }^{(5)} T_{A B},
$$

in which, the capital Latin indices run from zero to four, ${ }^{(5)} G_{A B}$ is $5 D$ Einstein tensor and ${ }^{(5)} T_{A B}$ is $5 D$ energy-momentum tensor. As an assumption, we consider ${ }^{(5)} T_{\alpha \beta}$ components of the ${ }^{(5)} T_{A B}$ to be the baryonic and dark matter source in a $4 D$ hypersurface, i.e. $T_{\alpha \beta}^{(M)}$, where the Greek indices go from zero to three. Hence, in this case we take ${ }^{(5)} T_{A B}=\operatorname{diag}\left(\rho_{M},-p_{M},-p_{M},-p_{M}, 0\right)$, in which $\rho_{M}$ and $p_{M}$ are respectively the energy density and the pressure of the matter (baryonic and dark matter). For cosmological purposes we restrict the $5 D$ metrics to the warped ones of the form, in local coordinates $x^{A}=\left(x^{\mu}, y\right)$,

$$
d S^{2}={ }^{(5)} g_{\mu \nu}\left(x^{C}\right) d x^{\mu} d x^{\nu}+g_{44}\left(x^{C}\right) d y^{2} \equiv g_{\mu \nu} d x^{\mu} d x^{\nu}+\epsilon b^{2}\left(x^{C}\right) d y^{2},
$$

where $y$ represents the fifth coordinate and $\epsilon^{2}=1$. Hereupon, after some manipulations, Eq. (2) on the hypersurface $\Sigma_{\circ}$ can be written as

$$
G_{\alpha \beta}=8 \pi G\left(T_{\alpha \beta}^{(M)}+T_{\alpha \beta}^{(X)}\right),
$$

where $T_{\alpha \beta}^{(X)}$ is considered as dark energy component of the energy-momentum tensor [34].

For a $5 D$ universe with an extra space-like dimension in addition to the three usual spatially homogenous and isotropic ones, metric (3), as a generalized FLRW solution, can be written as [34]

$$
d S^{2}=-d t^{2}+a^{2}(t) l^{2}(y)\left[\frac{d r^{2}}{1-k r^{2}}+r^{2}\left(d \theta^{2}+\sin ^{2} \theta d \varphi^{2}\right)\right]+b^{2}(t) d y^{2} .
$$

In general, we consider the scale factors to be functions of cosmic time and extra dimension coordinate. But, for mathematical simplicity, we assume that they are separable functions of cosmic time and extra dimension coordinate. Besides, the functionality of the scale factor of the fifth dimension on the extra dimension coordinate can be eliminated by transforming to a new extra coordinate.

By assuming $H \equiv \dot{a} / a, B \equiv \dot{b} / b$ and $L \equiv l^{\prime} / l$ and considering metric (5), the Einstein Eqs. (2) reduce as follows [34];

$$
H^{2}=\frac{8 \pi G}{3} \tilde{\rho}-\frac{k}{a^{2} l^{2}}
$$




$$
\frac{\ddot{a}}{a}=-\frac{4 \pi G}{3}(\tilde{\rho}+3 \tilde{p})
$$

and

$$
\frac{\ddot{a}}{a}=-H^{2}+\frac{1}{\alpha^{2} a^{2}} L^{2}-\frac{k}{a^{2} l^{2}},
$$

with

$$
B=H,
$$

which gives $b(t)=\left(b_{\circ} / a_{\circ}\right) a(t) \equiv \alpha a(t)$. Throughout this paper the subscript ' $\circ$ ' is used to indicate the present value of the quantities. In Eqs. (6) and (7) we have defined $\tilde{\rho} \equiv \rho_{M}+\rho_{X}$ and $\tilde{p} \equiv p_{M}+p_{X}$ with energy density and pressure of dark energy as

$$
\rho_{X} \equiv T_{t t}^{(X)}=\frac{3}{8 \pi G}\left[\frac{1}{\alpha^{2} a^{2}}\left(L^{\prime}+2 L^{2}\right)-H^{2}\right]
$$

and

$$
p_{X} \equiv-T_{i i}^{(X)}=\frac{1}{8 \pi G}\left[\dot{H}+3 H^{2}-\frac{1}{\alpha^{2} a^{2}}\left(2 L^{\prime}+3 L^{2}\right)\right] \equiv w_{X} \rho_{X} .
$$

Since the detailed coupling form among the matter and dark energy is unclear, one can expect their conservation equations may not to be independent. Hence, the total energy conservation equation,

$$
\dot{\tilde{\rho}}+3 H(\tilde{\rho}+\tilde{p})=0 .
$$

can plausibly separate into two distinguished equations for $\rho_{X}$ and $\rho_{M}$ as

$$
\dot{\rho}_{X}+3 H\left(\rho_{X}+p_{X}\right)=f(t)
$$

and

$$
\dot{\rho}_{M}+3 H\left(\rho_{M}+p_{M}\right)=-f(t),
$$

where $f(t)$ is assumed to be the interacting term between dark energy and matter.

By some manipulation, from Eqs. (13), (6) and (8) (see Ref. [34]) one gets

$$
f(t)=H \rho_{M},
$$

and

$$
L=\sqrt{C} \quad \text { and } \quad L^{\prime}=0,
$$

which gives $l(y)=l_{\circ} e^{ \pm \sqrt{C}\left(y-y_{\circ}\right)}$ where $C$ is a positive real constant. Eq. (15) emphasizes that in this model, matter and dark energy do interact with each other.

By substituting Eq. (6) in (8) and considering (16), one has

$$
\frac{\ddot{a}}{a}=-\frac{4 \pi G}{3} \rho_{M}-\frac{k}{2 l_{\circ}^{2} a^{2}} .
$$

Eq. (17) illustrates that, the geometrical term in acceleration equation is capable to make the universe expansion accelerates, when, universe is open. However, the measurements of anisotropies in the cosmic microwave background suggest that the ordinary $4 D$ universe is very close to a spatially flat one [35]-[38], but it does not mean the universe is exactly spatially flat. Recently, it has been shown that the observed large-scale cosmic microwave anomalies, discovered by WMAP and confirmed by the Planck satellite, are most naturally explained in the context of a marginally-open universe [39]. Also, there are several approaches, based on the string theory or quantum cosmology, in favor of the open universe [40]-[41]. Therefore sine now, we concentrate on the spatially open universe, i.e. $k=-1$.

Combination of Eqs. (14) and (15) gets

$$
\rho_{M}=\rho_{M \circ}\left(\frac{a_{\circ}}{a}\right)^{4}
$$




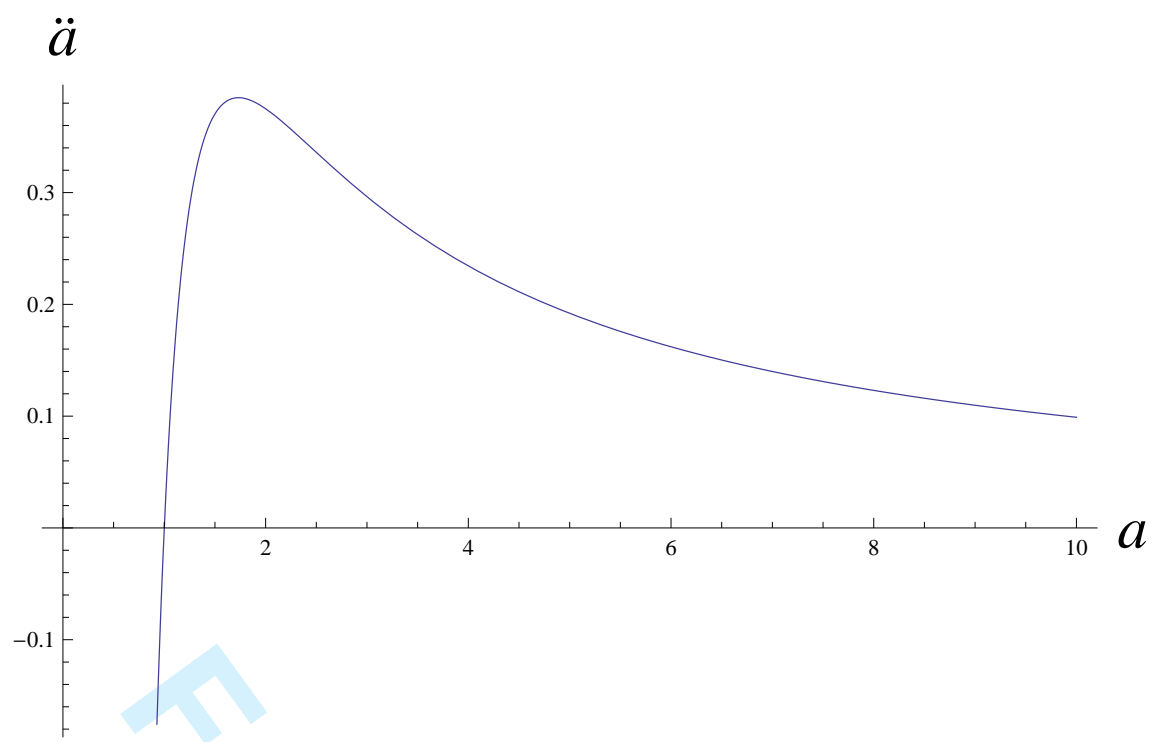

Figure 1: Changes of universe acceleration with evolution of the scale factor of ordinary spatial dimensions. For better show, the scales of the axes has been resized by taking $4 \pi G \rho_{M \circ} / 3 \equiv 1 \equiv 2 l_{\circ}$, in Eq. (19) which gives $\ddot{a}=0$ when $a=a_{\circ}^{2} l_{\circ}\left(8 \pi G \rho_{M \circ} / 3\right)^{1 / 2} \equiv 1$.

thus, Eq. (17) becomes

$$
\frac{\ddot{a}}{a}=-\frac{4 \pi G}{3} \rho_{M \circ}\left(\frac{a_{\circ}}{a}\right)^{4}+\frac{1}{2 l_{\circ}^{2} a^{2}},
$$

which shows that by the universe expansion the density of matter falls off more rapidly than the geometrical term. Hence, the matter dominating (deceleration) epoch in early time cannot last for ever and dark energy, i.e. geometrical term, (acceleration) finally comes to dominate. However, at the end, when scale factor $a$ becomes very very large, $\ddot{a}=0$ and universe will expand freely. See Table 1 and Fig. 1.

By comparing Eqs. (7)-(11) and (17) and some manipulations one can get the state parameter of dark energy, $w_{X}$ as

$$
w_{X}=-\frac{1}{3}\left(1+\frac{1}{l_{\circ}^{2}\left(2 C a_{\circ}^{2} / b_{\circ}^{2}-\dot{a}^{2}\right)}\right) .
$$

While after the Big Bang in classical limit, the speed of universe expansion was too high therefore,

\begin{tabular}{|c|c|c|}
\hline Universe Phase & $\ddot{a}$ & $a$ \\
\hline \hline \multirow{2}{*}{ Deceleration } & $\ddot{a} \leq 0$ & $0<a \leq a_{\circ}^{2} l_{\circ}\left(8 \pi G \rho_{M \circ} / 3\right)^{1 / 2}$ \\
\hline & $0<\ddot{a}<\ddot{a}_{M a x}$ & $a_{\circ}^{2} l_{\circ}\left(8 \pi G \rho_{M \circ} / 3\right)^{1 / 2}<a<a_{\circ}^{2} l_{\circ}\left(8 \pi G \rho_{M \circ}\right)^{1 / 2}$ \\
Accceleration & $\ddot{a}=\ddot{a}_{M a x}=\frac{1}{3} a_{\circ}^{-2} l_{\circ}^{-3}\left(8 \pi G \rho_{M \circ}\right)^{-1 / 2}$ & $a=a_{\circ}^{2} l_{\circ}\left(8 \pi G \rho_{M \circ}\right)^{1 / 2}$ \\
& $\ddot{a}_{M a x}>\ddot{a}>0$ & $a_{\circ}^{2} l_{\circ}\left(8 \pi G \rho_{M \circ}\right)^{1 / 2}<a<\infty$ \\
\hline
\end{tabular}

Table 1: Deceleration and acceleration epochs of the universe expansion and corresponding scale factor of ordinary spatial dimensions. 
according to the relation (20), the limit of state parameter was, $w_{X}=-1 / 3$. Then, matter domination caused universe expansion to slow down until $\ddot{a}=0$ when, $a=a_{\circ}^{2} l_{\circ}\left(8 \pi G \rho_{M_{\circ}} / 3\right)^{1 / 2}$ and $w_{X}=$ $-C a_{\circ}^{2} l_{\circ}^{2} / 3\left(C a_{\circ}^{2} l_{\circ}^{2}-b_{\circ}^{2}\right)$. After that, by the dark energy domination, $\ddot{a}>0$, which according to the Fig. 1 and Table 1 has a maximum, $\ddot{a}_{M a x}=1 / 3 a_{\circ}^{2} l_{\circ}^{3}\left(8 \pi G \rho_{M \circ}\right)^{1 / 2}$ at $a=a_{\circ}^{2} l_{\circ}\left(8 \pi G \rho_{M \circ}\right)^{1 / 2}$ with $w_{X}=-\left(3 C a_{\circ}^{2} l_{\circ}^{2}+b_{\circ}^{2}\right) / 3\left(3 C a_{\circ}^{2} l_{\circ}^{2}-2 b_{\circ}^{2}\right)$. Since then, the universe acceleration becomes small and small and finally get zero which causes universe expands freely when, $w_{X}=-\left(2 C a_{\circ}^{2} l_{\circ}^{2}+b_{\circ}^{2}\right) / 3\left(2 C a_{\circ}^{2} l_{\circ}^{2}-b_{\circ}^{2}\right)$.

At the end, we should remind that our approach in this manuscript is different from the brane world scenarios [42]- [46] and is based on the idea of induced matter theory, which have been applied for a non-vacuum five-dimensional version of general relativity, to introduce a geometrical interpretation for dark energy. In another word, in this model, the source of dark energy in the $4 D$ space-times can be viewed as a manifestation of extra dimension which imposes a geometrical term containing the intrinsic curvature, i.e. $k$, in the acceleration equation. Such a mechanism do not exist in the brane models.

\section{Conclusions}

It is a general belief that, what usually called dark energy makes the universe expansion to accelerate. Though, an enormous amount of work has been performed to explain this acceleration, but in many, the origin and the nature of dark energy is unclear yet.

Following the approach of the induced matter theory, we have investigated the cosmological implications of a non-vacuum five-dimensional version of general relativity in order to explain both decelerating and accelerating eras of the universe expansion. In this regard, on a $4 D$ hypersurface, we have classified the energy-momentum tensor into two parts. One part represents all kind of the matter (the baryonic and dark) and the other one contains every extra terms emerging from the fifth dimension, and has been considered as dark energy. Afterwards, in a $5 D$ space-time we have considered a generalized FLRW metric and derived the FLRW type equations and also energy conservation equation on the $4 D$ hypersurface.

Investigating the cosmological equations shows that matter (baryonic and dark) and dark energy interact with each other and also the geometry of the universe contributes in acceleration equation. The consistency of the model with observations imposes that the universe must be spatially open. Generally, the model is capable to explain respectively the decelerated expansion in early time and then accelerated expansion in late time of the universe evolution. However, it also predicts, this acceleration has a maximum value and after that decreases slowly to zero at infinity which, makes the universe expands freely at last.

\section{Acknowledgements}

We would like to thank Prof. Tim M.P. Tait at University of California, Irvine, for reading this article and useful comments.

\section{References}

[1] S. Perlmutter et al., Nature 391, 51 (1998).

[2] A.G. Riess et al., Astron. J. 116, 1009 (1998).

[3] S. Perlmutter et al., Astrophys. J. 517, 565 (1999). 
[4] P. de Bernardis et al., Astrophys. J. 564, 559 (2002).

[5] S.M. Carroll, Living Rev. Rel. 4, 1 (2001).

[6] S.M. Carroll, "Why is the Universe Accelerating?", astro-ph/0310342.

[7] V. Sahni, Lect. Notes Phys. 653, 141 (2004).

[8] E.J. Copeland, M. Sami and S. Tsujikawa, Int. J. Mod. Phys. D 15, 1753 (2006).

[9] M. Kamionkowski, "Dark Matter and Dark Energy", astro-ph/0706.2986.

[10] J. Frieman, M. Turner and D. Huterer, Ann. Rev. Astron. Astrophys. 46, 385 (2008).

[11] S. Tsujikawa, "Dark energy: investigation and modeling", astro-ph/1004.1493.

[12] J. Yoo, "Theoretical Models of Dark Energy", astro-ph/1212.4726.

[13] I. Zlatev, L. Wang and P.J. Steinhardt, Phys. Rev. Lett. 82, 896 (1999).

[14] A. Albrecht and C. Skordis, Phys. Rev. Lett. 84, 2076 (2000).

[15] M.C. Bento, O. Bertolami and N.C. Santos, Phys. Rev. D 65, 067301 (2002).

[16] D. Blais and D. Polarski, Phys. Rev. D 70, 084008 (2004).

[17] C. Armendariz-Picon, V. Mukhanov and P.J. Steinhardt, Phys. Rev. Lett. 85, 4438 (2000).

[18] R.J. Scherrer, Phys. Rev. Lett. 93, 011301 (2004).

[19] A.Y. Kamenshchik, U. Moschella and V. Pasquier, Phys. Lett. B 511, 265 (2001).

[20] M.C. Bento, O. Bertolami and A.A. Sen, Phys. Rev. D 66, 043507 (2002).

[21] J.E.M. Aguilar, C. Romero and A. Barros, Gen. Rel. Grav. 40, 117 (2008).

[22] J. Ponce de Leon, Class. Quant. Grav. 27, 095002 (2010).

[23] J. Ponce de Leon, JCAP 03, 030 (2010).

[24] S.M. M. Rasouli, M. Farhoudi and H.R. Sepangi, Class. Quant. Grav. 28, 155004 (2011).

[25] A.F. Bahrehbakhsh, M. Farhoudi and H. Shojaie Gen. Rel. Grav. 43, 847 (2011).

[26] A. F. Bahrehbakhsh, M. Farhoudi, and H. Vakili, Int. J. Mod. Phys. D 22, 1350070 (2013).

[27] G. Nordstrøm, Phys. Z. 15, 504 (1914).

[28] T. Kaluza, Sitz. Preuss. Akad. Wiss. 33, 966 (1921).

[29] O. Klein, Z. Phys. 37, 895 (1926).

[30] J.M. Overduin and P.S. Wesson, Phys. Rep. 283, 303 (1997).

[31] P.S. Wesson, Space-Time-Matter, Modern Kaluza-Klein Theory (World Scientific, Singapore, 1999).

[32] P.S. Wesson, Five-Dimensional Physics (World Scientific, Singapore, 2006).

[33] M. Pavšič, "The Landscape of Theoretical Physics: A Global View from Point Particles to the Brane World and Beyond, in Search of a Unifying Principle" gr-qc/0610061. 
[34] A.F. Bahrehbakhsh, "Interacting Induced Dark Energy Model" gr-qc/1601.00310v1.

[35] N.A. Bachcall et al., Science 284, 1481 (1999).

[36] P. de Bernardis et al., Nature 404, 955 (2000).

[37] S. Hanany et al., Astrophys. J. 545, L5 (2000).

[38] A. Balbi et al., Astrophys. J. 545, L1 (2000).

[39] A.R. Liddle, M. Cortes, Phys. Rev. Lett. 111, 111302 (2013).

[40] M. A. Dariescu and C. Dariescu, Astrophys. Space Sci. 361, 6, 195 (2016).

[41] D.H. Coule, J. Martin, Phys.Rev. D 61, 063501 (2000).

[42] L. Randall and R. Sundrum, Phys. Rev. Lett. 83, 3370 (1999).

[43] I. Brevik and A. Hallanger, Phys. Rev. D 69, 024009 (2004).

[44] K. Ghoroku, M. Tachibana and N. Uekusa, Phys. Rev. D 68, 125002 (2003).

[45] M. D. Maia, E. M. Monte and J. M. F. Maia, Phys. Lett. B 585, 11 (2004).

[46] R. Maartens and K. Koyama, Living Rev. Rel. 13, 5 (2010) 